\title{
Selectivities in carbometalation of olefins. Experimental and theoretical studies
}

\author{
Eiichi Nakamura \\ Department of Chemistry, University of Tokyo, Hongo, Bunkyo-ku, Tokyo 113, Japan
}

\begin{abstract}
Despite the potential synthetic utility of the reaction, the elements that control the regio- and stereochemistry of the addition of an organometallic reagent to an olefin have not received careful attention. Our recent studies on the selectivities in organometallic addition to cyclopropenone acetals have revealed some principles of regio- and stereocontrol in organometallic additions to olefins. As guided by the computational analysis of the experimental selectivities, it was found that the controlling elements are quite different from those known for organometallic additions to carbonyl compounds.
\end{abstract}

\section{Introduction: basic problems in olefin carbometalation reaction}

The addition of an organometallic to a simple olefin is a reaction of great synthetic potential in that the reaction creates two contiguous chiral centers on a planar olefinic bond in a single step (eq 1). However, the potential of this reaction in the synthesis of enantio- and diastereomerically pure compounds have been little explored, and various fundamental questions remained yet to be addressed.

Addition of an organometallic (R-M) to an unactivated olefin (eq 1) is generally difficult to control since such an addition may lead to polymerization of the olefin unless the initial adduct is stabilized in some way. (Note that the conjugate addition of organocuprates to $\alpha, \beta$-unsaturated carbonyl compounds may be viewed mechanistically as an extension of carbonyl addition rather than olefin carbometalation.) In order to obtain a single, enantiomerically and diastereomerically pure product by carbometalation of an unsymmetrical olefin, the reaction must first be regioselective. This problem does not exist in the carbonyl addition. Sizable experimental data on regioselectivity of carbometalation of olefins and acetylenes have been accumulated, but it was only recent that the mechanistic understanding of such selectivity was achieved on a solid theoretical basis. ${ }^{1}$

Once the regiochemistry is defined, selection of the olefinic face becomes the next issue. Whereas diastereoface selectivity (e.g., Cram's rule) has been studied extensively in the carbonyl addition, much less has been examined for the olefinic version. ${ }^{2}$ Ligand controlled enantioface selective addition to a simple olefins has not been studied either.

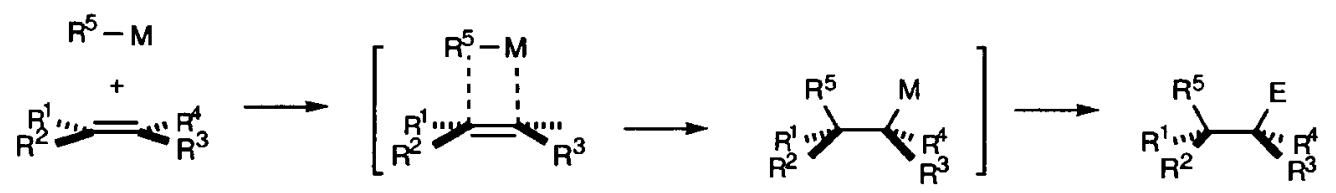

When one adds an allylic metal compound to an olefin (eq 2, sometimes called "metallo-ene reaction"), there arises an additional problem of mutual face selectivity, which is reflected in the 1,2-simple diastereoselectivity with regard to the newly formed $\mathrm{C}-\mathrm{C}$ bond. In the carbonyl addition of allyl metal and enolate anion, this issue is usually reduced to the selectivity involving chair and boat transition states. In the olefinic reaction, however, it is unclear whether or not the same chair/boat protocol also operates.

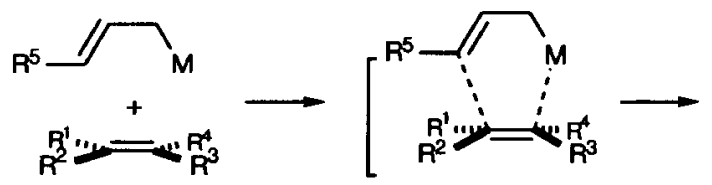

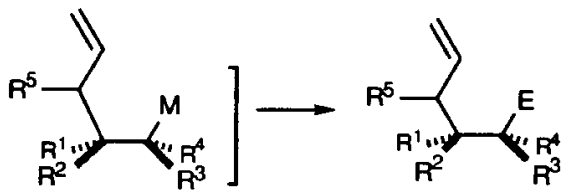


For the olefin carbometalation reaction to be practically useful, all these issues should be resolved, ideally from both mechanistic and practical viewpoints: unfortunately, there has been little information, especially on the issues of regio-, enantio- and diastereoface selectivity. In the past several years, we have been engaged in the studies of carbometalation of olefins, learned basic principles that govern the selectivities of olefin carbometalation, and developed some synthetically useful transformations. In the following paragraphs, we will summarized the results from experimental and theoretical viewpoints.

\section{Selective carbometalation of cyclopropenes}

We sometime ago made two interesting findings that helped us address the questions on the selectivities of carbometalation of olefins. First, the development of efficient synthetic routes to substituted cyclopropenone acetals (CPAs) was instrumental for the present studies. Thus, commercially available 1,3-dichloroacetone was acetalized (97\%) and then was treated with three equivalents of $\mathrm{NaNH}_{2}$, which effects cyclization and deprotonation of the acidic vinyl proton in a single pot to generate a sodium salt (a) in $>85 \%$ yield (eq 3 ). Subsequent addition of an alkyl halide afforded a substituted CPA in high overall yield. Generation and electrophilic trapping of the lithium (b) and zinc salts (c) allowed the preparation of various other derivatives $\left(R=\right.$ aryl, vinyl, etc.). ${ }^{3}$ Chiral acetals have been prepared in the same manner.<smiles>CC1(C)COC(CCl)(CCl)OC1</smiles>

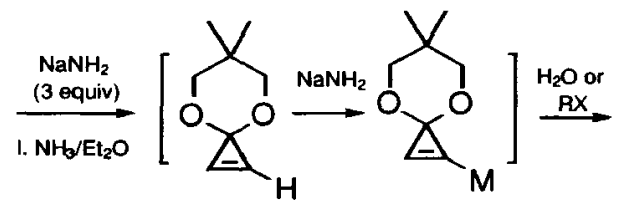<smiles>[R]C1CC12OCC(C)(C)CO2</smiles>

a: $M=N a, b: M=L i, c: M=Z n(I I)$

The second key observation was that various organocopper reagents add rapidly to CPAs in high yield, 4 suggesting that this chemistry provides an opportunity to study various aspects of reactivity and selectivity of olefin carbometalation reactions (Scheme 1). 5 The addition of $\mathrm{Me}_{2} \mathrm{CuLi}$ to the parent $\mathrm{CPA}$ at $-78^{\circ} \mathrm{C}$ was instantaneous giving the methylated adduct ( $R^{1}=\mathrm{Me}$, in $96 \%$ yield as determined after methanol quench). Deuterium quench and MeI quench indicated that the carbocupration took place in a cis fashion. A variety of homo- and heterocuprates afforded cyclopropyl cuprates, which were then trapped by a variety of electrophiles (Scheme 1). The scheme also illustrates some useful applications to the synthesis of 1,4-keto esters, cyclopentenes, cycloheptadienes, and dienones. 6

Scheme 1
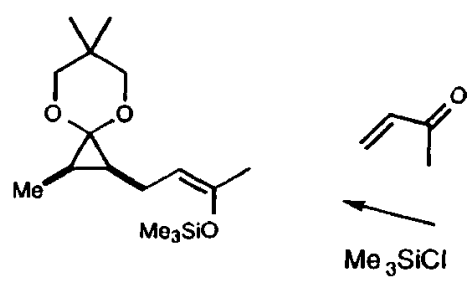

$\left(R^{1}=\mathrm{Me}\right)$

PhCOCI<smiles>[X]C(=O)C(CC(=O)c1ccccc1)CC(=O)c1ccccc1</smiles>

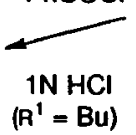

$\mathrm{X}=\mathrm{OCH}_{2} \mathrm{CMe}_{2} \mathrm{CH}_{2} \mathrm{OH}$
Mel $\left(R^{1}=\right.$ hexenyl)<smiles>CC1(C)COCOC1</smiles>

$160^{\circ} \mathrm{C}$<smiles>C=CC1(/C=C\CBr)OCC(C)(C)CO1</smiles>

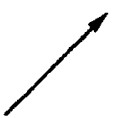

$\mathrm{H}^{+}$

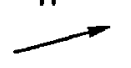

Bu
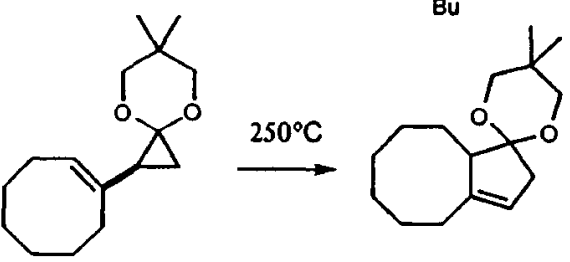

$\left(R^{\prime}=\right.$

cyclooctenyl)
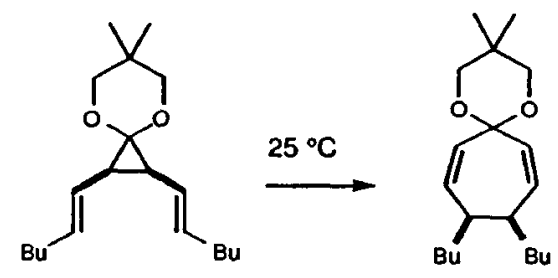

cat. $\mathrm{Pd}$

( $R^{1}=$ hexenyl)

(C) 1996 IUPAC, Pure and Applied Chemistry 68, 123-130 


\section{1,4-Asymmetric induction}

Experiments: The foregoing findings set the stage to study the diastereoface selectivity of the carbocupration of homochiral acetals (eq 4). We initially found that the addition of dialkyl cuprates to the parent chiral CPA at $-70^{\circ} \mathrm{C}$ showed very moderate stereoselectivity (ca. $70 \%$ d.s.) to give the carbocupration product. However, the reaction of a bulkier "higher order" cuprate showed satisfactory selectivity (96\%d.s.), ${ }^{7}$ and this selectivity profile suggested that the reaction takes place in such a manner that a bulkier group is oriented toward the equatorial carbon of the cyclopropene ring in the transition state shown below.

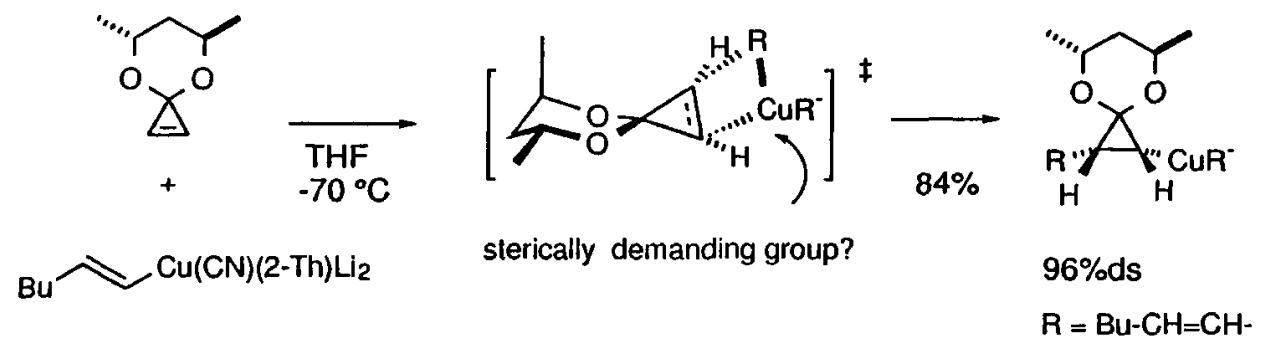

The regioselectivity in the reaction of the 2-substituted cyclopropenes (eq 5) was very high (29:1-99:1), favoring the formation of the 2,2-dialkyl adduct so that the reaction places the copper atom on the less sterically hindered position. With respect to 1,4-asymmetric induction, we found much higher selectivity of 90:10 96:4, yet surprisingly with selectivity opposite to the one mentioned above. The reasons for this reversal of the stereoselectivity were unclear at the beginning. However, the theory (vide infra) suggests that the selectivity might well reflect the product stability.
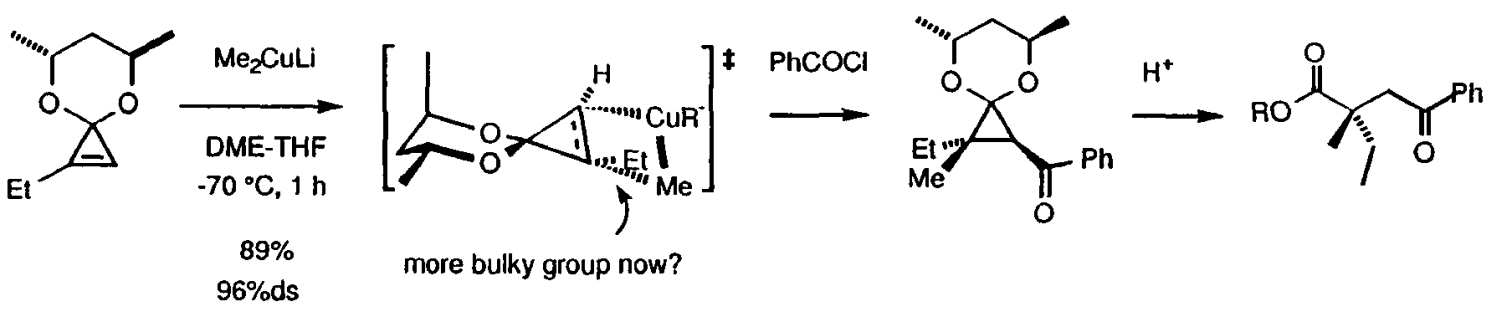

Theoretical analysis: A simplistic mechanistic view of the carbometalation of cyclopropenes suggests that the transition state of this highly exothermic reaction may be reactant-like. However, attempted rationalization of the above experimental results turned out to invoke a late transition state. Systematic comparison of the reaction of $\mathrm{Me}^{-}, \mathrm{MeLi}$, and $\mathrm{MeCu}$ with ethylene and cyclopropene led to the following conclusions about the reaction course of the carbometalation of cyclopropene. ${ }^{8}$

In consonance with the high exothermicity of the reaction, the $\mathrm{Me}^{-}$and $\mathrm{MeLi}$ additions to cyclopropenes involve rather early transition states. In the $\mathrm{MeCu}$ addition to cyclopropene, however, the cyclopropene moiety in the transition state experiences considerable rehybridization (namely, product-like in the cyclopropane region and advanced $\mathrm{C}-\mathrm{Cu}$ bond formation). Therefore, the reaction takes place from the less hindered side with respect to the chiral acetal group to eventually place the bulkier group on the equatorial cyclopropenyl carbon.

\section{Mutual face selection in allylmetalation reaction: chair or boat transition state?}

Experiments: We next investigated the diastereo- and enantio selectivity of allylmetalation of CPAs. The first point of interest was whether the reaction conforms to the usual chair/boat protocol so often invoked in the analysis of mutual face selectivity in the carbonyl addition of allylic metal and metal enolate species. 9 
For the reaction between the CPA and allylic zinc reagents shown in eq 6 , we investigated the issue of $\mathrm{C}_{3}-\mathrm{C}_{4}$ diastereoselectivity-an issue rarely examined in the carbometalation of olefins. The reaction with a zinc reagent prepared from transcinnamyl bromide and zinc metal in THF took place slowly at $0{ }^{\circ} \mathrm{C}$ to give the adduct with $64 \%$ d.s.. Replacement of the bromide ligand with a bulky electron donor ligand not only accelerated the addition, but also improved selectivity. Thus, mixed reagents prepared by treatment of cinnamylzinc bromide with one equivalent of either 2,4,6trimethylphenyllithium reacted with at $-23{ }^{\circ} \mathrm{C}$ with $81-89 \%$ d.s Addition of HMPA (2 equiv) further improved the selectivity to $95-96 \%$ d.s. In all cases, the allylic group (rather than the mesityl dummy ligand) was transferred selectively to the cyclopropene with virtually complete $\mathrm{S}_{\mathrm{E}} 2$ '-regioselectivity $(>97 \%)$.
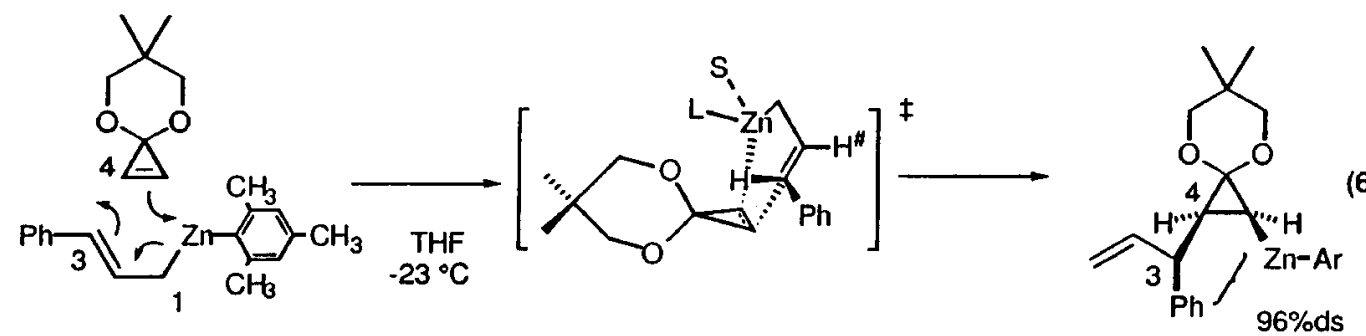

Theoretical analysis: The stereoselectivities of allylzincation can be controlled by suitable choice of ligands. The selectivity could be explained by assuming a twist chair transition state (in eq 6), where the acetal and the vinylic proton $\left(\mathrm{H}^{\#}\right)$ are oriented away from each other. However, the reason why such a model is successful in accounting for the selectivity was by no means clear, since no information was available for the transition state of such reactions. To address this issue, we first examined the simplest prototype, the $\mathrm{SE}_{\mathrm{E}}$ '-addition of allylzinc chloride and allylmagnesium chloride to acetylene. As shown below, the transition structures (TSs) obtained at the HF/3-21G-ECP level were virtually identical for the two different metals. The most striking feature of these TSs, as compared to those of the allylmetalation of a carbonyl compound, is the planar arrangement of the nucleophilic allylic end, two acetylenic carbons, and the metal electrophile. This planarity may be due to two electronic constraints: conjugation between the $\mathrm{C}=\mathrm{C}$ and $\mathrm{Zn}-\mathrm{C}$ bonds in the allylic zinc reagent, and the spatial location of the $\mathrm{C}_{3}, \mathrm{C}_{4}, \mathrm{C}_{5}$ and the $\mathrm{Zn}$ atoms lying nearly on the same plane. The latter is due to the importance of $\pi$ and $\pi^{*}$ orbitals in carbometalation of olefins as opposed to $n$ and $\pi^{*}$ in carbonyl additions. 10
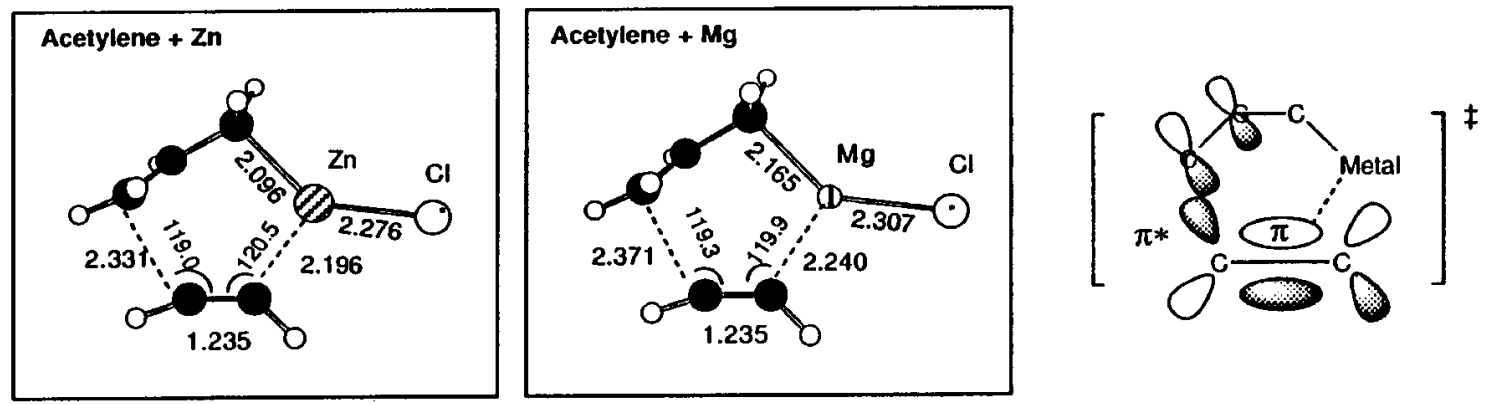

We then proceeded to examine more realistic models, namely, the $\mathrm{S}_{\mathrm{E}} 2$-selective addition of $(E)-\mathrm{RCH}=\mathrm{CHCH}_{2} \mathrm{ZnCl}\left(\mathrm{H}_{2} \mathrm{O}\right)\left(\mathrm{R}=\mathrm{H}\right.$ and $\left.\mathrm{CH}_{3}\right)$ to cyclopropene at the $\mathrm{HF} / 6$ 31G*//HF/3-21G-ECP level. Two isomeric TSs $\mathbf{A}^{1}$ and $\mathbf{A}^{2}$ of nearly equal energies that are responsible for the $\mathrm{C}_{3}-\mathrm{C}_{4}$ diastereoselectivity were obtained, and some important characteristics were noted. First, only the half-chair TSs are available for the reaction. Second, because of this arrangement, there occurs torsional strain for the forming $\mathrm{C}_{3}-\mathrm{C}_{4}$ bond in $\mathbf{A}^{1}$ (indicated by an arrow) which may slightly destabilize this TS. In the TS $\mathbf{A}^{2}$, on the other hand, the short distance $(2.593 \mathrm{~A}$ ) between the two asterisked hydrogens suggests that $\mathbf{A}^{2}$ would be significantly destabilized if $\mathrm{H}^{* *}$ were replaced by an alkoxy group as in our actual substrates 1 . Third, the coordination environment around the zinc atom does not 
drastically change the essential structural features of the twist-chair TS (as revealed by the calculations in the presence and the absence of coordinated water). This information now sets the stage for exploration of ligand-induced asymmetric carbometalation reactions.

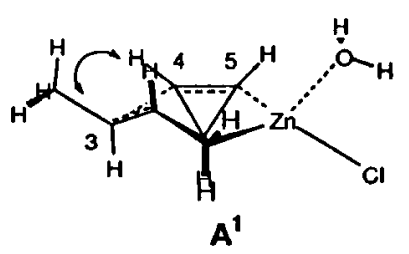

$(0.369 \mathrm{kcal} / \mathrm{mol})$

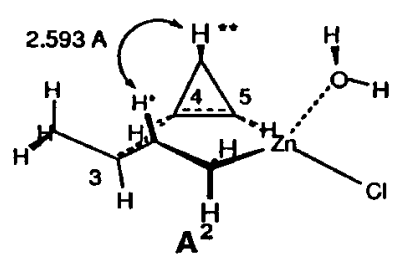

$(0.0 \mathrm{kcal} / \mathrm{mol})$

(HF/6-31G*//HF/3-21G energies)

\section{Enantioselective allymetalation}

Experiments: Enantioselective allylmetalation of an olefin is generally a difficult synthetic operation and has not been studied thus far. The issue of product selectivity in this reaction is much more complicated than the carbonyl addition since the reaction creates three chiral centers in one step. The symmetrical cis structure that simplifies the otherwise complicated regio- and stereochemical problem. Although we examined, in vain, a variety of chiral amino alcohols, which are effective for carbonyl addition of dimeric zinc reagents, we eventually found that the chiral bisoxazoline (BOX) ligands (see below) ${ }^{11}$ give excellent enantioselectivity (eq 7).12 The chiral reagents were several times more reactive than the parent allylic zinc bromide reagent.

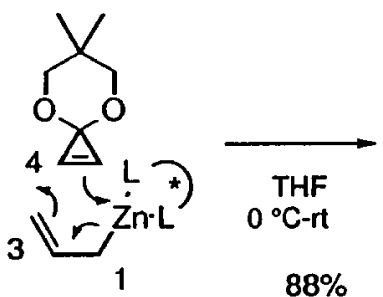

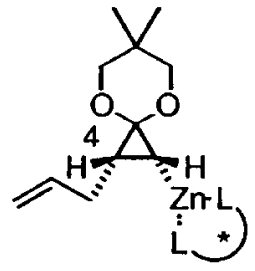

$\mathrm{C}_{4}=R, 96 \%$ ee

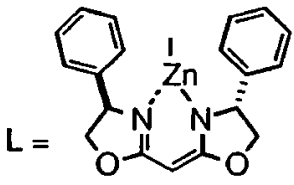

The chiral BOX ligand ( $\mathrm{R}^{\prime}=\mathrm{Ph}$, eq 7 ) derived from $R$-phenylglycine was lithiated (at -78 to $0^{\circ} \mathrm{C}$ in THF), and treated with allyzinc bromide (from allyl bromide and activated zinc) at $0^{\circ} \mathrm{C}$ to room temperature. The chiral reagent $\left(R=H, R^{\prime}=P h\right)$ was then reacted with CPA at room temperature for several hours. Quenching with $\mathrm{NH}_{4} \mathrm{Cl}$ afforded the product $R-\mathrm{C}_{4}$ in $85 \%$ yield with $96 \%$ e.e. The chiral allyl- and prenyl zinc reagents derived from $S$-valine gave the antipodes and with $>98 \%$ and $93 \%$ e.e., respectively (eq 8 ).

We then examined the reaction of trans-substituted allylic zinc reagents $(\mathrm{R}=\mathrm{Me}, \mathrm{Ph})$ (eq 8): the sense of enantioselection was the same as found for the parent allylzinc reagent and the mutual face selectivity was the same as for the achiral reagents. However, the levels of the selectivities were uniformly lower. The $\mathrm{C}_{3}-\mathrm{C}_{4}$ diastereoselectivity was only moderate $\left(73 \%\right.$ d.s. and $83 \%$ d.s. for $R=M e, R^{\prime}={ }^{i} \operatorname{Pr}$ and $R=c-H e x, R^{\prime}={ }^{i} \operatorname{Pr}$, respectively). Though the enantioselectivity also suffered from the substitution, it was improved to $97 \%$ e.e. by the use of a bulkier $\mathrm{BOX}$ ligand with $\mathrm{R}={ }^{t} \mathrm{Bu}$. This ligand, however, also failed to improve the $\mathrm{C}_{3}-\mathrm{C}_{4}$ selectivity ( $81 \%$ d.s.).

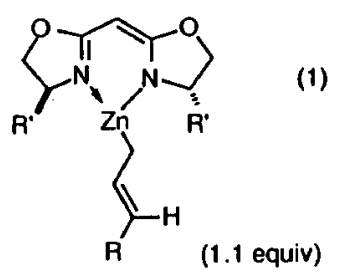

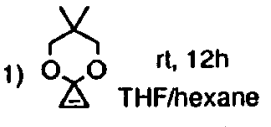

(2) sat. $\mathrm{NH}_{4} \mathrm{Cl}$

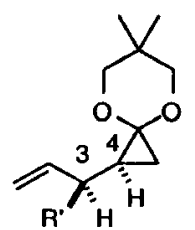

major diastereomer
(8)

\begin{tabular}{ccccc} 
R & R' $^{\prime}$ & \%yield & ds & es \\
\hline Me & $i P r$ & 81 & $73: 27$ & $>98: 2$ \\
C-Hex & $i$ Pr & 94 & $83: 17$ & $82: 18$ \\
C.Hex & $t-B u$ & 58 & $81: 19$ & $98.5: 1.5$
\end{tabular}


Theoretical analysis: While an empirical stereochemical model ${ }^{13}$ proved insufficient, theoretical analysis provided valuable information on the function of the chiral ligand in determining the enantio- and diastereoselectivities. With the MNDO hamiltonian, we found four diastereomeric TSs for the reaction of the BOX zinc reagent the BOX zinc reagent $\left(\mathrm{R}=\mathrm{H}, \mathrm{R}^{\prime}={ }^{i} \mathrm{Pr}\right)$ with the parent CPA lacking the acetal gem-dimethyl group. The lowest energy TS in Figure 1 (corresponding to the attack B). The acetal group is fitted into the "cleft" (curved lines) formed by the two isopropyl groups of the ligand. The second lowest energy TS $(0.32 \mathrm{kcal} / \mathrm{mol}$ higher in energy) is due to the approach of the cyclopropene from the top side (heavy arrow $\mathbf{C}$ ) with the acetal orientation as indicated. The two alternative approaches (light arrows $\mathbf{D}$ and $\mathbf{E}$ ) are higher in energy (1.13 and 1.69 $\mathrm{kcal} / \mathrm{mol}$, respectively). These approaches are stereochemically degenerate: both $\mathbf{B}$ and $\mathbf{C}$ produce one enantiomer, and $\mathbf{D}$ and $\mathbf{E}$ another. The calculated energetics thus qualitatively reproduces the experimental selectivity.

Unlike a chair TS which is conformationally well defined, the twisted chair in this carbometalation reaction is rather flexible as suggested by the very small $B / C$ energy difference of $0.32 \mathrm{kcal} / \mathrm{mol}$ (vide supra). In summary, the BOX ligand recognizes the global enantiotoposity of the TS with its asymmetric cleft, but cannot control the local conformation of the TS inside this cleft. The issue of global vs. local controls is likely to be wide spread among ligand controlled stereoselective reactions.

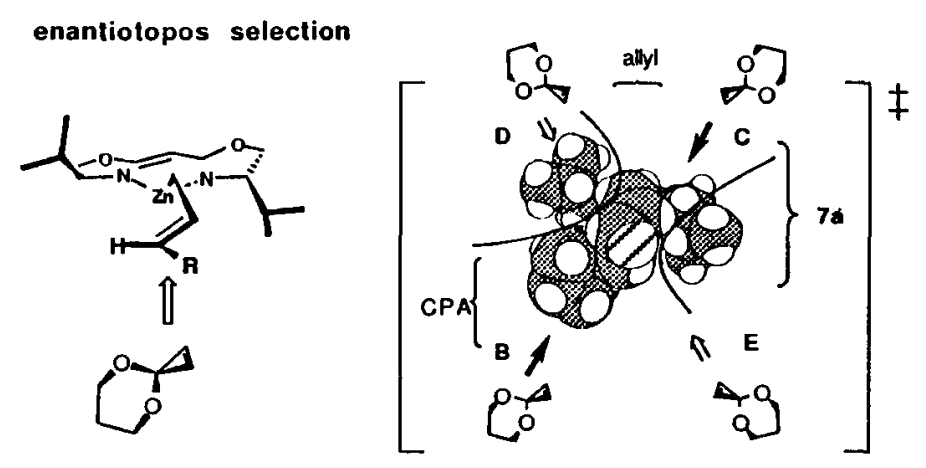

Figure 1.

Upon closing this account, we note an observations about diastereo- and enantioselectivities that we made during the theoretical studies on the reaction pathways (rather than the static TSs of organometallic addition reactions (Scheme 2). As noted above, the allylmetalation of an olefin takes place via a single TS type, namely, a twistchair form (Scheme $2 \mathrm{~A}$ ) since both the $\pi$ and $\pi^{*}$ orbitals share the same symmetry plane perpendicular to the olefinic face. On the other hand, the allylmetal addition to a carbonyl group (or the aldol reaction) takes place either via chair or boat TS (Scheme $2 B$ ). This is due to the orbital arrangement where the electrophilic $\pi^{*}$ orbital and the basic lone pair orbital which serves as an anchor for metal complexation are perpendicular to each other. This orbital arrangement determines important general features of organometallic additions to carbonyl groups, which characterize the transition state as well as the overall reaction pathway of the carbonyl addition reactions. 14,15

Another interesting consequence of the difference in the orbital orientations between carbonyl compounds and olefins is its effects on the enantioface selection. In the olefin addition, the initial metal/ $\pi$ complex is already chiral (Scheme 2A), and hence an entropically unfavorable bimolecular dissociation/re-association is necessary to achieve an alternative enantioface selectivity. In the carbonyl addition, however, only an intramolecular bond rotation is needed for attaining face selective addition enantioface selection, as the initial metal/lone pair complex is essentially achiral (Scheme $2 B$ ). While the actual energy cost of such difference obviously depends on the reaction, it seems interesting to speculate that the carbonyl face selection, which is essentially the selection of rotational mode upon conversion from the metal/lone complex to the TS, is more facile than the olefinic face selection. Even a cursory inspection of the recorded data on enantioface 
A

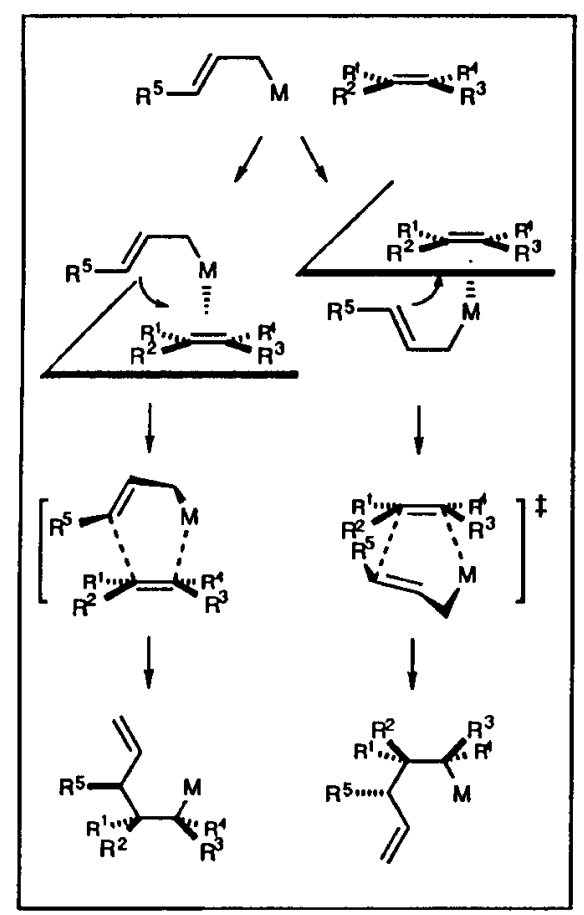

B

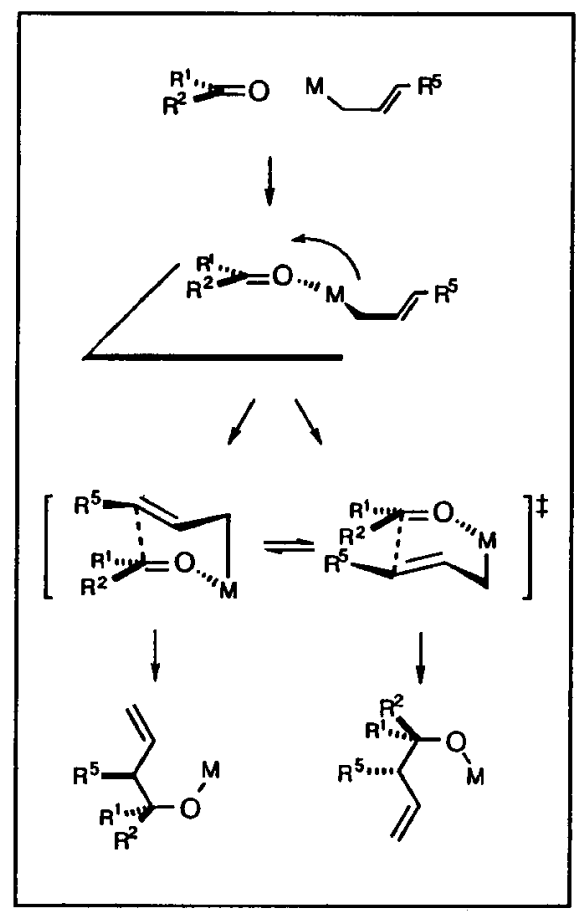

Scheme 2. Allylmetalation of $\mathrm{C}=\mathrm{C}$ and $\mathrm{C}=\mathrm{O}$

selective reactions suggests that succeful examples of olefinic enantioface selection requires more elaborate and bulkier ligand than those employed for carbonyl enantioface selection. One possible way to bypass dissociation/re-association problem in the olefin reactions may be the use of a substrate possessing an anchor group which makes the initial metal complex effectively achiral. The well known substrates of such a kind are allylic alcohols and $\alpha, \beta$-unsaturated carbonyl compounds.

A series of experiments described above demonstrated that the selectivities of carbometalation reaction can be controlled by suitable choice of auxiliary, ligand and reaction conditions. Theoretical analyses have proven to be useful for understanding the experimental data, designing ligand and reaction conditions, and clarification of the difference between the carbonyl and the olefin additions.

Acknowledgment. The author deeply appreciates the collaboration with the coworkers whose names appear in the references, and Prof. K. Morokuma for collaborative work in theoretical analyses of the reactions, which immensely expanded our imagination on the nature of chemical reactions. Financial support from the Ministry of Education, Culture, and Science, Japan is gratefully acknowledged.

\section{References}

1. E. Nakamura, Y. Miyachi, N. Koga, and K. Morokuma, J. Am. Chem. Soc. 1146686 (1992).

2. M. Arai, T. Kawasuji, and E. Nakamura, J. Org. Chem. 58, 5121 (1993).

3. M. Isaka, S. Matsuzawa, S. Yamago, S. Ejiri, Y. Miyachi, E. Nakamura, J. Org. Chem. 54, 4727 (1989). M. Isaka, S. Ejiri, and E. Nakamura, Tetrahedron, Symposium in Print 48, 2045 (1992). E. Nakamura, J. Synth. Org. Chem. Jpn. 52, 935 (1994).

4. E. Nakamura, M. Isaka, and S. Matsuzawa, J. Am. Chem. Soc. 1101297 (1988).

5. $\quad$ E. Nakamura, Synlett, 539 (1991).

6. E. Nakamura, K. Kubota, and M. Isaka, J. Org. Chem. 57, 5809 (1992). K. Kubota, M. Isaka, and E. Nakamura, Heterocycles, in press. 
7. M. Isaka and E. Nakamura, J. Am. Chem, Soc., 1127428 (1990).

8. E. Nakamura, M. Nakamura, Y. Miyachi, N. Koga, and K. Morokuma, J. Am. Chem. Soc. 11599 (1993).

9. K. Kubota, M. Nakamura, M. Isaka, and E. Nakamura, J. Am. Chem. Soc. 1155867 (1993).

10. M. Nakamura, E. Nakamura, N. Koga, and K. Morokuma, J. Chem. Soc., Faraday Trans. 29, 17890 (1994).

11. R. E. Lowenthal, A. Abiko, S. Masamune, Tetrahedron Lett. 31, 6005 (1990). D. A. Evans, K. A. Woerpel, M. M. Hinman, M. Faul, J. Am. Chem. Soc. 113726 (1991). E. J. Corey, N. Imai, H.-Y. Zhang, J. Am. Chem. Soc. 113728 (1991). H. Fritschi, U. Leutenegger, A. Pfaltz, Angew. Chem., Int. Ed. Engl. 25, 1005 (1986).

12. M. Nakamura, M. Arai, and E. Nakamura, J. Am. Chem. Soc. 1171179 (1995).

13. Cf. Pfaltz, A. Acc. Chem. Res. 1993,26, 1005.

14. M. Nakamura, E. Nakamura, N. Koga, K. Morokuma, J. Am. Chem. Soc. 11511016 (1993).

15. S. Mori, M. Nakamura, E. Nakamura, N. Koga, and K. Morokuma, J. Am. Chem. Soc. 117, 5055 (1995). 